\title{
IDBA - A Practical Iterative de Bruijn Graph De Novo Assembler
}

\author{
Yu Peng, Henry C.M. Leung, S.M. Yiu, and Francis Y.L. Chin \\ Department of Computer Science, The University of Hong Kong \\ Pokfulam Road, Hong Kong \\ \{ypeng, cmleung2, smyiu, chin\}@cs.hku.hk
}

\begin{abstract}
The de Bruijn graph assembly approach breaks reads into $k$-mers before assembling them into contigs. The string graph approach forms contigs by connecting two reads with $k$ or more overlapping nucleotides. Both approaches must deal with the following problems: false-positive vertices, due to erroneous reads; gap problem, due to non-uniform coverage; branching problem, due to erroneous reads and repeat regions. A proper choice of $k$ is crucial but for single $k$ there is always a trade-off: a small $k$ favors the situation of erroneous reads and non-uniform coverage, and a large $k$ favors short repeat regions.

We propose an iterative de Bruijn graph approach iterating from small to large $k$ exploring the advantages of the in between values. Our IDBA outperforms the existing algorithms by constructing longer contigs with similar accuracy and using less memory, both with real and simulated data. The running time of the algorithm is comparable to existing algorithms.
\end{abstract}

Availability: IDBA is available at $h t t p: / / w w w . c s . h k u . h k / \sim a l s e / i d b a /$

Keywords: De novo assembly, de Bruijn graph, string graph, mate-pair, high throughput short reads.

\section{Introduction}

Despite tremendous research efforts, de novo assembly remains an only partially solved problem. Although more reference genomes are known for efficient resequencing, de novo assembly remains a critical step in studying a genome. Applications such as detection of structural variations [1] cannot be done easily based on resequencing techniques and there are evidences that show genome assembly based on resequencing may produce errors especially for species with high mutation rates [1].

With high throughput sequencing technologies (e.g. Ilumina Genome Analyzer and Applied Biosystems SOLiD), mate-pair short reads (35nt to $75 \mathrm{nt}$ ) of a mammalian genome can be generated in a few weeks at low cost. As short reads have different characteristics (i.e. shorter length, higher coverage, but relatively higher error rates) when compared to traditional Sanger reads, new assembly tools have emerged [2-11]. The first batch of tools (e.g. SSAKE [3], VCAKE [4], SHARCGS [5]) uses the "overlap-then-extend" idea but need to rely on data structures such as prefix trees, so they require lots of memory and run very slowly. The newer tools are divided into those based on the de Bruijn graph (e.g. Velvet [7], Abyss [8], Euler-SR[2, 6], 
AllPaths[11]) and those based on the string graph (e.g. Edena [9]). Each of the two approaches has merits and limitations, and it is not clear which is better.

De Bruijn graph algorithms [7-8, 12-13] assemble reads by constructing a de Bruijn graph in which each vertex represents a length- $k$ substring ( $k$-mer) in a length- $l$ read and connects vertex $u$ to vertex $v$ if $u$ and $v$ are consecutive $k$-mers in a read, i.e. the last $(k-1)$ nucleotides of the $k$-mer represented by $u$ is the same as the first $(k-1)$ nucleotides of the $k$-mer represented by $v$. Intuitively, maximal paths of vertices without branches in the graph correspond to contigs to be outputted by algorithms.

String graph algorithms $[9,14]$ represent each read by a vertex and there is a directed edge from vertex $u$ to vertex $v$ if the suffix of at least $x$ nucleotides of read $u$ is the same as the prefix of read $v$. The value of $\mathrm{x}$ is the number of overlapping nucleotides for two consecutive reads. Similar to de Bruijn graph algorithms, string graph algorithms report maximal paths without branches as a contigs.

When the reads are error-free with high coverage, most tools work well. However, because of repeats, erroneous reads, and non-uniform coverage, their performances is not always acceptable. In this paper, we focus on three major problems: (1) false positive vertices (due to errors in reads); (2) gap problem (due to non-uniform or low coverage) and; (3) branching problem (due to repeats or errors in reads).

\section{Three major problems}

(a) False Positive Vertices: Errors in reads introduce false positive vertices which make both graphs bigger and consume more memory; for example, for the human genome with 30x coverage, the memory requirement of Velvet [7] and Abyss[8] is more than $250 \mathrm{G}$.

(b) Gap problem: Due to non-uniform or low coverage, reads may not be sampled for every position in the genome. For the de Bruijn graph, when all the (possible $l-k$ ) reads covering consecutive $k$-mers are missing, we may have short "dead-end" paths. The larger the $k$, the more serious is the gap problem. The same applies to the string graph if all the (possible $l-x$ ) reads following another read are missing.

(c) Branching problem: Those $k$-mers which connect with multiple $k$-mers due to repeat regions or erroneous reads introduce branches in the de Bruijn graph. Many algorithms [7-9] stop the contigs at branches and it is not possible to extend a contig without additional information. A small $k$ will lead to more branches. The same branching problem occurs in string graph algorithms, and depending on the value of $x$, the same read can be connected with multiple other reads.

\section{Existing assembly algorithms}

Table 1 summarizes the major techniques used by existing algorithms to solve the above problems. There are two methods for handling false positive vertices. (1) Deadend removal: False positive vertices usually lead to short dead-end paths. Both de Bruijn and string algorithms (e.g. [7-8]) remove false positive vertices by removing these paths. However, due to the gap problem, some paths may be removed by mistake. (2) Filtering: de Bruijn graph algorithms remove false positive vertices if the corresponding $k$-mers appear no more than $m$ times. However, some correct $k$-mers with low coverage might also be removed especially for large $k$ for which the expected $k$-mer occurrence frequency is low. As for string graph algorithms, the 
expected occurrence of each read is also low (1 or 2) and they rely on error correction which falls back to the multiplicity of k-mers [15] to correct errors in each read before forming contigs.

Table 1. Major techniques used to handle the three problems

\begin{tabular}{|c|c|c|c|}
\hline Problems & Techniques & de Bruijn graph & String graph \\
\hline \multirow{2}{*}{$\begin{array}{l}\text { 1) False positive } \\
\text { vertices }\end{array}$} & (i) Dead-end removal & Yes & Yes \\
\hline & (ii) Filtering & $\begin{array}{l}\text { Yes (not effective } \\
\text { if } k \text { is large) }\end{array}$ & $\begin{array}{l}\text { Not applicable (relies on } \\
\text { error correction algorithms) }\end{array}$ \\
\hline 2) Gap & $\begin{array}{l}\text { No effective method (try to } \\
\text { use a reasonable small } k \text { or } x \text { ) }\end{array}$ & -- & -- \\
\hline \multirow[t]{2}{*}{ 3) Branching } & (i) Using read information & Yes & $\begin{array}{l}\text { Not applicable (already use } \\
\text { the whole read information) }\end{array}$ \\
\hline & (ii) Bubble removal & Yes & Yes \\
\hline
\end{tabular}

There is no effective method to deal with the gap problem except all algorithms try to avoid gaps by using a small $k$ (or $x$ in string graphs).

Some de Bruijn graph algorithms [12] solve the branching problem by considering only those branches that are supported by reads. However, this method may easily lead to erroneous contigs [12] if the reads are erroneous especially when error rates are high. This method cannot be applied to string graph algorithms as they already consider the read information. The other technique is bubble removal, which is used by both approaches [7-9] and tries to merge similar paths of very similar vertices into one path as the small differences may only be due to SNPs or errors. However, the merging might be incorrect and this process increases the length of contigs at the expense of their accuracy.

Table 2. Performance (N50) of three existing assembly algorithms (Edena, Velvet, Abyss) against IDBA under different coverage and error rates for the simulated dataset using E.coli where read length is $75 \mathrm{nt}$. The best results generated are used for comparison.

\begin{tabular}{lcccc}
\hline & $\begin{array}{c}\text { High Coverage } \\
\text { Low Error Rate } \\
(100 x, 0.5 \%)\end{array}$ & $\begin{array}{c}\text { Low Coverage } \\
\text { Low Error Rate } \\
(30 \mathrm{x}, 1 \%)\end{array}$ & $\begin{array}{c}\text { High Coverage } \\
\text { High Error Rate } \\
(100 \mathrm{x}, 2 \%)\end{array}$ & $\begin{array}{c}\text { Low Coverage } \\
\text { High Error Rate } \\
(30 \mathrm{x}, 2 \%)\end{array}$ \\
\hline Edena (string Graph) & 63256 & 5104 & 53491 & 147 \\
Velvet (de Bruijn Graph) & 63214 & 24772 & 59285 & 16527 \\
Abyss (de Bruijn Graph) & 58678 & 22109 & 50009 & 10992 \\
IDBA (our algorithm) & 63218 & 63218 & 59287 & 32612 \\
\hline
\end{tabular}

To summarize, string graph algorithms do not have an effective method to remove errors from reads and have the gap problem if $x$ is set to a reasonable value to avoid the branching problem. However, string graph algorithms, which make use of the direct information in the whole read, perform very well in case of high coverage and low error rate. For other cases, de Bruijn graph algorithms may perform better. Our observations are confirmed by the N50 comparison of Edena [9] (currently one of the best string graph algorithms), Velvet [7] and Abyss [8] (the best de Bruijn graph algorithms) based on different coverage and error rates of the data as shown in Table 2 (more details on the comparison can be found in Section 4). 
The best existing assembly algorithms are Edena (string graph based), Velvet and Abyss (both de Bruijn graph based, differing in the exact details for handling deadends and bubble removal). However, setting the correct parameter $k$ in de Bruijn graph algorithms (or $x$ in string graph algorithms) is crucial. The k parameter (or $\mathrm{x}$ ) affect the filtering and, moreover, provides a trade-off between the gap problem and the branching problem. In order to minimize the number of gaps, a smaller $k$ (or $x$ ) should be used. But with a small $k$ (or $x$ ), the branching problem becomes more serious. Existing algorithms usually pick a moderate value for $k$ (or $x$ ) to balance between the two problems. None of the existing approaches try to take advantage of using different $k$ (or $x$ ) values ${ }^{1}$.

\section{Our contributions}

We propose a new assembly algorithm (IDBA) based on the de Bruijn graph. The idea is simple but practical in that it alleviates the difficulties in setting a correct $k$ and the filtering threshold $m$, gives good results, uses much less memory (many existing tools require huge amount of memory making them impractical for large genomes) at the expense of a reasonable increase in running time. Instead of using a fixed $k$, our algorithm iterates from small to large $k\left(k_{\min }\right.$ to $\left.k_{\max }\right)$ capturing the merits of all values in between. The key step is to maintain an accumulated de Bruijn graph to carry useful information forward as $k$ increases. Note that this is not the same as running the algorithm for many different $k$ values independently as it is not clear how to combine contigs from different runs to get a better result. We show theoretically that the accumulated de Bruijn graph can capture good contigs and these contigs can be made longer as $k$ increases. Based on experiments on simulated and real data, we show that IDBA can produce longer contigs (see Table 2 for the N50 comparison) with similar accuracy (very few wrong contigs and high coverage). More detailed results are presented in Section 4.

We are able to reduce the memory consumption by $50-80 \%$ as compared to existing algorithms which use a fixed $k$ of moderate size. Because $k$ is of moderate size, the algorithms cannot do filtering in the first step especially when the coverage is not high and thus create a big graph due to false positive vertices. However, since IDBA starts with a small $\mathrm{k}$, many false positive vertices are pruned with a conservative and effective filtering in the first step (e.g., set $\mathrm{m}=1$ ). Although IDBA iterates through different $k$ values, with implementation tricks (described in Section 2), IDBA runs a lot faster than Abyss and is comparable with other existing algorithms.

\section{Organization of the paper and remarks}

We organize the paper as follows. In Section 2, we introduce our algorithm IDBA and show the advantages of using small and large $k$ values. Also, we provide key implementation details which help to reduce the memory consumption and running time. Section 3 compares the performance of IDBA with existing algorithms on both simulated and real data. We conclude the paper in Section 4.

\footnotetext{
${ }^{1}$ The SHARCGS [5] algorithm uses fixed $x$ values (the number of overlapping nucleotides) when extending a read, but they repeat the whole assembling procedure independently using a few different $x$ values and combine the resulting contigs from different runs only.
} 
We note that using mate-pair information to resolve repeats that are longer than reads is another important aspect of an assembly tool. In this paper, we mainly focus on short repeats, which account for the largest portion of repeats in genomes and cannot be resolved by mate-pair information easily as the variation of the insert size may be even larger than the length of the repeat. We leave the problem of how to use mate-pair information in assembly more effectively for future study. Hence, the last step of our assembly tool, which uses mate-pair information to connect the contigs, simply follows Abyss [8]. Note also that, although our approach can be applied to the string graph with a range of $x$ values, currently there is no effective way to remove errors from reads for string graphs, and so we focus on de Bruijn graphs.

\section{Algorithm IDBA}

Given a set of reads, we denote the de Bruijn graph for any fixed $k$ as $G_{k}$. Instead of using only one fixed $k$, IDBA (Iterative de Bruijn Graph short read Assembler) iterates on a range of $k$ values from $k=k_{\min }$ to $k=k_{\max }$ and maintains an accumulated de Bruijn graph $H_{k}$ at each iteration. In the first step, $k=k_{\min }, H_{k}$ is equivalent to the graph $G_{k}$ after deleting all vertices whose corresponding $k$-mers appear no more than $m$ times (we set $m=1$ or 2 in practice depending on the coverage of the input reads) in all reads. Theorem 3(in the Appendix) shows that these $k$-mers are very likely to be false positives.

To construct $H_{k+1}$ from $H_{k}$, we first construct potential contigs in $H_{k}$ by identifying maximal paths $v_{1}, v_{2}, \ldots, v_{p}$ in which all vertices have in-degree and out-degree equal to 1 except $v_{1}$ and $v_{p}$ which may have in-degree 0 and out-degree 0 , respectively. Note that a path of $p$ vertices represents a potential contig of length $p+k-1$. We remove all reads that can be represented by potential contigs in $H_{k}$ i.e. those reads that are substrings of a contig (as these reads cannot be used to resolve any branch). In the construction of $H_{k+1}$, we only consider the remaining reads and the potential contigs in $H_{k}$. We perform two steps to convert $H_{k}$ to $H_{k+1}$. (1) For each edge $\left(v_{i}, v_{j}\right)$ in $H_{k}$, we convert the edge into a vertex (representing a $(k+1)$-mer $\left.x_{i 1} x_{i 2} \ldots x_{i k} x_{j k}=x_{i 1} x_{j 1} \ldots x_{j k}\right)$. (2) We connect every two such vertices by an edge if the corresponding two consecutive $(k+1)$-mers have support from one of the remaining reads or potential contigs of $H_{k}$, i.e. the corresponding $(k+2)$-mer exists.

Note that in practice, we do not need to go from $k$ to $k+1$; we can jump from $k$ to $k+s$, in which case, for (1), we convert each path of length $s$ in $H_{k}$ into a vertex. In Theorem 5 in the Appendix, we show that by setting $s=1$, we may get high quality contigs. As $s$ increases, we expect the quality of contigs will drop, so it is always better to use a small $s$. The choice of $s$ will represent a trade-off on the efficiency of the algorithm and the quality of the contigs.

For each $H_{k}$, we follow other algorithms [7] to remove dead-ends (potential contig shorter than $3 k-1$ with one end with 0 in-degree or out-degree, which represents a path in $H_{k}$ of length at most $2 k$ ). Note that removing a dead-end may create more dead-ends, the procedure will repeat until no more dead-ends exist in the graph. These dead-end contigs are likely to be false positives (to be discussed in the Appendix). In fact, most of the remaining false positive vertices after the first filtering step can be removed as dead ends and the accuracy of the contigs produced by IDBA is high. 
After obtaining $H_{k_{\max }}$, we merge bubbles where bubbles are two paths representing two different contigs going from the same vertex $v_{1}$ to the same vertex $v_{p}$ where these two contigs differ by only one nucleotide. This scenario is likely to be caused by an error or a SNP. Like other assembly algorithms [7-9], we merge the two contigs into one. We base on mate-pair information to connect the contigs as much as possible by using a similar algorithm as Abyss[8] and report the final set of contigs.

\section{Algorithm IDBA}

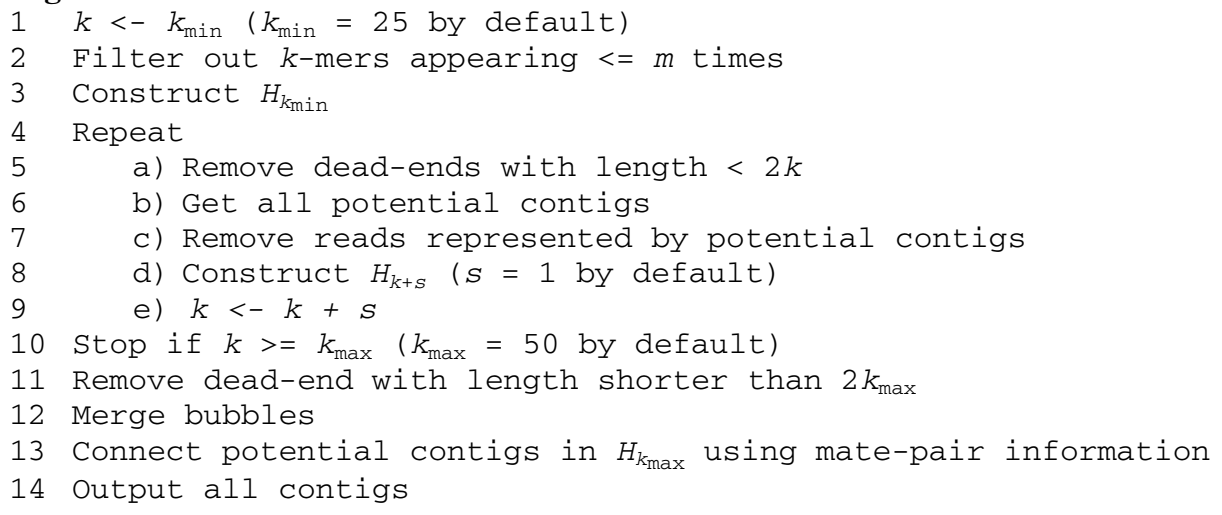

Note that the probability of removing a true positive vertex in our filtering step is very low (Theorem 3 in Appendix A.3 gives the analysis) as long as $k_{\min }$ and the filtering threshold $m$ are set to a reasonable value (e.g. $m=1$ ). For example, if $1.6 \times 10^{6}$ length-75 reads are sampled from a genome of length $4.1 \times 10^{6}$ (45x coverage) with error rate $1 \%$, the probability of filtering out a true positive vertex in $H_{25}$ is $1.14 \times 10^{-9}$, i.e. the expected number of false negative vertices is $0.0047<<1$ which is very small. Even for some cases where the expected number of false negative vertices is large, say 10, it is still relatively very small when compared with the genome size. Thus, for simplicity in analysis, we assume there is no false negative vertex in $H_{k_{\min }}$. The filtering step can remove a large portion of the false positive vertices. Most of the remaining false positive vertices are removed in later steps by dead-ends The probability of removing a correct contig as a dead-end is also small (see Theorem 4 in Appendix A. 3 for the exact calculation of the probabilities). The probability of determining a dead-end wrongly is only $2.46 \times 10^{-4}$ when the above example is considered.

Due to the gap problem a contig that appears in $G_{k}$, for a small k, might not be a contig in $G_{k^{\prime}}$ for $k^{\prime}>k$. However, in IDBA, if a contig $c$ appears in $H_{k}$, there must be a contig $c^{\prime}$ in $H_{k}$ containing $c$ (Theorem 1). That is, the contig information is carried over from $H_{k}$ to $H_{k}$. As $k$ increases, more branches can be resolved while the gaps solved when $k$ is small in previous iterations will be preserved.

Theorem 1: Assume that $k_{\min }=k$ and $k<k^{\prime}$. If there is a contig $c$ in $G_{k}$ of length at least $3 k_{\max }-1$ with all true positive vertices, there must be a contig $c^{\prime}$ in $H_{k}$, such that $c^{\prime}$ contains $c$. 
Proof: By induction on $k$. Let $k^{\prime}=k+1$ and $c=x_{1} x_{2} \ldots x_{p+k-1}$ be a contig in $H_{k}$ represented by the path $p=\left(v_{1}, v_{2}, \ldots, v_{p}\right)$, all vertices $v_{1}, v_{2}, \ldots, v_{p}$ have in-degree and out-degree $\leq 1$, it is easy to see that the path $p^{\prime}=\left(v_{1}, v_{2}, \ldots, v_{p-1}{ }^{\prime}\right)$ in $H_{k+1}$ where each $(k+1)$-mer $v_{i}^{\prime}=x_{i} x_{i+1} \ldots x_{i+k}$ also has in-degree and out-degree $\leq 1$. As the length of the contig represented by path $p^{\prime} \geq 3 k_{\max }-1$, there must be a contig including path $p$, i.e. $c$, in $H_{k+1}$.

Corollary: $H_{k_{\max }}$ must contain all contigs in $G_{k_{\min }}$ of length at least $3 k_{\max }-1$ with all true positive vertices.

In practice $H_{k_{\max }}$ always contains longer contigs than $G_{k_{\min }}$ by resolving branches at each iteration. As Figure 1 shows, by iterating the graph $H_{k}$ towards larger $k$, we may get longer and longer contigs as some of the branches (e.g. length- $k$ repeat region (Case 1) and error branches in $H_{k+1}$ (Case 2)) may be resolved when using a larger $k$.

Case 1:
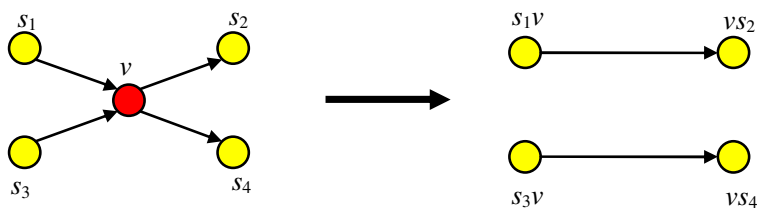

Case 2:
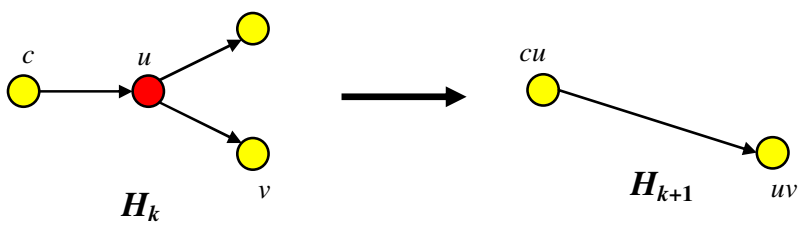

Fig. 1. Two cases for having longer contigs

Case 1: Let $c_{1}=s_{1} v_{r} s_{2}$ and $c_{2}=s_{3} v_{r} s_{4}$ be two substrings in the genome where $v_{r}$ is a common length- $k$ substring representing a repeat region, $s_{1}, s_{2}, s_{3}, s_{4}$ are different substrings. $c_{1}$ and $c_{2}$ are represented by five contigs in $H_{k}$ as the $k$-mer $v_{r}$ has in-degree of 2 and out-degree of 2 . If there are two correct reads containing $v_{r}$ and its 2 neighboring nucleotides at both ends in $c_{1}$ and $c_{2}$ respectively, and there is no error read containing $s_{1} v_{r} s_{4}$ or $s_{3} v_{r} s_{2}$, then there must be two contigs, one containing $c_{1}$ and the other containing $c_{2}$ in $H_{k+1}$.

Case 2: Let $c$ be a contig in $H_{k}$ that stops before vertex $u$ whose in-degree is 1 and out-degree is $>1$. Assume that among all branches of $u$, only $u$ to $v$ is correct. If there is a correct read containing $u$ and its 2 pairs of neighboring nucleotides at both ends and there is no error read linking $c$ with other branches, there will be a longer contig $c^{\prime}$ in $H_{k+1}$ that contains $c$.

Case 1 and Case 2 prove the following theorem.

Theorem 2: If there is a contig $c$ in $G_{k}$ of length at least $3 k_{\max }-1$ with all vertices are true positive which satisfies case 1 or case 2 in $H_{k}, k=k_{\min } \leq k^{\prime}<k_{\max }$, there is a longer contig $c^{\prime}$ in $H_{k_{\max }}$ that contains $c$. 
In the algorithm, we increase the value of $k$ by 1 at each iteration, i.e. $s=1$. Theorem 5 in Appendix A.3 shows that for a better quality of the contigs, this is essential. On the other hand, as a trade-off between the efficiency of the algorithm and the quality of the contigs, it is possible to set $s>1$, i.e. to increase the value of $k$ by more than 1 at each iterative step.

\subsection{Implementation Details}

The memory used by IDBA is only about $20-30 \%$ of that used by the other existing tools because $80 \%$ of false positive vertices are removed in the filtering step (line 2 in algorithm IDBA) and IDBA uses a compact hash table to represent de Bruijn graph implicitly with each edge represented by one bit only.

Although IDBA constructs $H_{k_{\max }}$ from $H_{k_{\min }}$ step by step, the running time of IDBA is not directly proportional to the number of $k$ values between $k_{\max }$ and $k_{\min }$. According to Theorem 1, a contig in $H_{k}$ is also a contig in $H_{k+1}$, thus IDBA only needs to check whether a branch in $H_{k}$ can be resolved in $H_{k+1}$. Since reads represented by a contig are removed in each iteration, the number of reads in each iteration decreased. In practice, about half of the reads are removed when constructing $H_{k_{\min +1}}$ and IDBA runs much faster than Abyss, and about three times slower than Velvet.

\section{Experimental Results}

The genome of Escherichia coli (O157:H7 str. EC4115) from NCBI [16] is used for simulated experiments (the genome length is $5.6 \mathrm{M}$ ). Reads are randomly sampled uniformly with coverage 30x. In our experiments, we generated reads with error rates $1 \%$, read length 75 and insert distance 250 . Note that we have repeated the experiments using other coverage (e.g. 50x, 100x), error rates (e.g. 2\%) and read length (e.g. 50). The results are similar, so we only show the result for $30 x$ coverage with $1 \%$ error on length-75 reads. We also use a real data set, namely Bacillus Subtilis, to evaluate our algorithm. The length of the genome is $4.1 \mathrm{M}$. The reads are sequenced using Solexa machine with coverage $45 x$, read length 75 and insert distance 400 . The estimated error rate is about $1 \%$.

\subsection{Simulated Data}

We compare the performance of Velvet ${ }^{2}$, Abyss $^{3}$, Edena and our algorithm IDBA on the simulated data based on different $k$ values (or $x$ values). For IDBA, we fix $k_{\min }=$ $25, m=1$ and compare the performance of IDBA with different $k_{\max }$. For the other algorithms, defaults parameters provided by the assemblers are used. We also plot the upper bound that can be achieved by building an ideal de Bruijn graph with no false positive or false negative vertices and edges and produce all single paths as contigs.

\footnotetext{
${ }^{2}$ Since pair-end version of Velvet performs not well and pair-end version of Abyss outperforms Velvet in the quality of results, we only show result of single-end version of Velvet.

${ }^{3}$ Since SHARCGS is too slow and Abyss applies a similar idea as Euler with better performance, we leave SHARCGS and Euler out of our comparison.
} 
We calculate N50 and coverage only for valid contigs which are longer than 100 bps and can be aligned to the reference with $99.9 \%$ similarity. Figure 2(a) shows the comparison of the algorithms based on N50. As we mentioned in the introduction (Section 1), existing assembly algorithms have many false positive vertices and branching problems when $k$ is small and they have many gaps when $k$ is large. Thus these algorithms have the best performance (largest N50) for in-between values of $k$ (the optimal $k$ for Velvet, Edena and Abyss are 35, 40 and 40 respectively in this data set). Since IDBA considers a range of $k$ values, its performance is better than the others even when considering a range of 10 values for $k\left(k_{\min }=25\right.$ and $\left.k_{\max }=35\right)$. Furthermore, when IDBA considers a larger range for $k\left(k_{\min }=25\right.$ and $\left.k_{\max }=50\right)$, its performance is close to the upper bound. We have only 10 false positive contigs when setting $k_{\min }=25$ and $k_{\max }=50$ while Abyass, Velvet and Edena produce 489, 19 and 650 false positive contigs respectively.

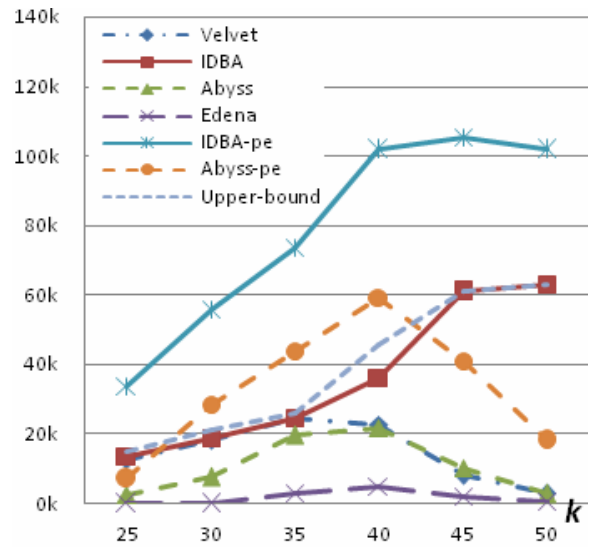

(a)

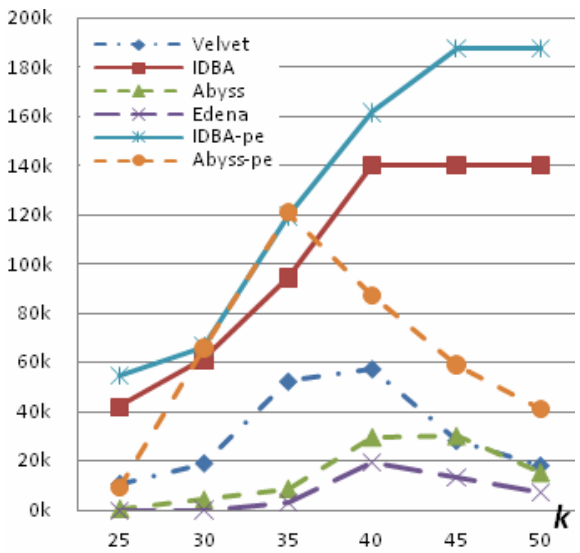

(b)

Fig. 2. (a) N50 for contigs produced by assembly algorithms with different $k$-values ( $x$-values if the software is string graph based) on simulated data using E.coli as the reference genome where read length is $75 \mathrm{nt}$, coverage is $30 \mathrm{x}$ and error rate is $1 \%$. (IDBA-pe and abyss-pe are the results for using mate-pair information to extend the contigs while Edena does not use matepair information) (b) N50 for contigs produced by assembly algorithms with different $k$ (or $x$ ) values on real data from bacillus subtilis where read length is $75 \mathrm{nt}$, coverage is $45 \mathrm{x}$ and error rate is $1 \%$.

For IDBA and Abyss, we also apply the mate-pair information to connect the resulting contigs to make them longer. The results are shown in the same graph (IDBApe and abyss-pe). Note that as $k$ increases, the N50 may drop when applying the mate-pair procedure since more branches have been resolved incorrectly and some short contigs are removed as dead-ends. In fact, further research is required on how to use mate-pair information effectively for assembly. The pair-end version of Abyss has optimal result when $k$ is 35 while IDBA has optimal result when $k$ is 45 . 
Table 3. Statistics of optimal (w.r.t. N50) result of each algorithm for simulated data

\begin{tabular}{|c|c|c|c|c|c|c|c|c|}
\hline & Time & Memory & $k$ & Number & N50 & $\begin{array}{l}\text { Contigs } \\
\text { Max length }\end{array}$ & False pos. contigs (total len.) & Coverage \\
\hline Velvet & $155 \mathrm{~s}$ & $1641 \mathrm{M}$ & 35 & 1412 & 24772 & 127265 & $70(35589)$ & $95.29 \%$ \\
\hline Edena & $957 \mathrm{~s}$ & $678 \mathrm{M}$ & 40 & 4672 & 5104 & 46908 & $650(72019)$ & $97.22 \%$ \\
\hline IDBA-pe & $412 \mathrm{~s}$ & $360 \mathrm{M}$ & $25-45$ & 709 & 105579 & 217365 & $43(164120)$ & $93.94 \%$ \\
\hline Abyss & $1114 \mathrm{~s}$ & $1749 \mathrm{M}$ & 40 & 1390 & 22109 & 87118 & $66(34998)$ & $95.05 \%$ \\
\hline
\end{tabular}

Table 3 shows a comprehensive statistics on the performance of the algorithms on their optimal $k$ values (w.r.t. N50). IDBA produced much longer contigs than all other algorithms. When mate-pair information is not available, the N50 of IDBA (63218) is about three times that of the next best algorithm ( 24772 by Velvet) and is the same as the upper bound. IDBA also produced the fewest number of wrong contigs (a contig which cannot be aligned to the reference genome with $99.9 \%$ similarity) and the total length of all wrong contigs is only about 4500nt which is much less than the other algorithms. The coverage of IDBA is also the best among all algorithms. Since IDBA performs well on assembling single end reads, it outperforms other algorithms even when use mate-pair information. To conclude, IDBA outperforms other algorithms substantially and produces much longer contigs with higher accuracy.

\subsection{Real Data}

Figure 2(b) shows the N50 of the contigs produced by Velvet, Abyss, Edena and our algorithm IDBA on the real reads from Bacillus Subtilis using different $k$ values ( $x$ values). Since the reads may not be uniformly sampled in the real data set, we use a smaller $k_{\min }(20 \mathrm{nt})$ and keep $m=1$ to run IDBA. For the other algorithms, we use their default parameters except for $k$. We do not have the reference genome to check if a contig is valid. We calculate the N50 for all reported contigs longer than 100bp. Note that the result may not be accurate, because some algorithms may produce longer but invalid contigs. The results are consistent with that of the simulated data. Velvet, Edena and Abyss get their best performance when $k=40,40$ and 45 respectively. IDBA can keep improving the result while $k_{\max }$ is increasing.

In this data set, mate-pair information is not so useful for IDBA because using read information can already solve most of the branches. When using $k_{\max }$ equal to 50 , the N50 pair-end version of IDBA produced is $30 \%$ longer than single end version. The performance of mate-pair version Abyss has similar performance as in simulated data. Its optimal $k$ is 35 , and the longest N50 it produces is even shorter than single end IDBA. In conclusion, IDBA produced the longest contigs among all algorithms. A detailed comparison is given in Table 4 in Appendix A.2.

\subsection{Running Time and Memory Consumption}

Other than Abyss (12.8 minutes - 7 hours for simulated data and 10 minutes - 1.2 hours for real data depending on the value of $k$ ), the running time of other algorithms are more or less the same. Abyss runs much slower when $k$ is small, probably due to its slow procedure for dealing with graphs with many false positive vertices. Velvet 
(120 - 220 seconds for simulated data and $130-200$ seconds for real data) is the fastest among all algorithms. IDBA (180 - 350 seconds for simulated data and 280 330 seconds for real data) runs faster than Abyss and is about three times slower than Velvet. Refer to Figures 3 and 4 in the Appendix for details.

The memory consumption is about the same for different $k$ values across the existing algorithms. Abyss and Velvet require about $2 \mathrm{G}$ bytes of memory for simulated data and $1 \mathrm{G}$ memory for real data. IDBA only requires about $400 \mathrm{M}$ and $300 \mathrm{M}$ respectively because $80 \%$ of false positive vertices are removed in the first filtering step. Note that only 8 25-mers are removed incorrectly in simulated data set (it matches with expected number 8.88 calculated in Theorem 3 ). So, the memory consumption of IDBA is only about $20-30 \%$ of the existing de Bruijn graph tools. Edena consumes less memory than Abyss and Velvet because the number of reads is small, but still double the size used by IDBA. Refer to Tables 3 in Section 3.2 and Table 4 in the Appendix for details.

\section{Conclusions}

Our IDBA algorithm, based on de Bruijn graphs, can capture the merits of all $k$ values in between $k_{\min }$ and $k_{\max }$ to achieve a good performance in producing long and correct contigs. Because the initial filtering step removes many false positive $k$-mers and the number of reads considered at each iterative step is reduced, the required memory and running time is much reduced. Though an accumulated de Bruijn graph is maintained at each iterative step, the running time is comparable with the existing algorithms. In fact, this running time can be further reduced if, say, one or two $k$ values are skipped at each iterative step. In practice, the quality of the result is only slightly affected by the skipping of values, in exchange for shorter running time.

Our next target is to investigate how to better use mate-pair information for resolving long repeats in order to produce even longer and more accurate contigs.

\section{References}

1. Wang, J., et al.: The diploid genome sequence of an Asian individual. Nature 456(7218), 60-65 (2008)

2. Chaisson, M.J., Brinza, D., Pevzner, P.A.: De novo fragment assembly with short matepaired reads: Does the read length matter? Genome Res. 19(2), 336-346 (2009)

3. Warren, R.L., et al.: Assembling millions of short DNA sequences using SSAKE. Bioinformatics 23(4), 500-501 (2007)

4. Jeck, W.R., et al.: Extending assembly of short DNA sequences to handle error. Bioinformatics 23(21), 2942-2944 (2007)

5. Dohm, J.C., et al.: SHARCGS, a fast and highly accurate short-read assembly algorithm for de novo genomic sequencing. Genome Res. 17(11), 1697-1706 (2007)

6. Chaisson, M.J., Pevzner, P.A.: Short read fragment assembly of bacterial genomes. Genome Res. 18(2), 324-330 (2008)

7. Zerbino, D.R., Birney, E.: Velvet: algorithms for de novo short read assembly using de Bruijn graphs. Genome Res. 18(5), 821-829 (2008)

8. Simpson, J.T., et al.: ABySS: a parallel assembler for short read sequence data. Genome Res. 19(6), 1117-1123 (2009) 
9. Hernandez, D., et al.: De novo bacterial genome sequencing: millions of very short reads assembled on a desktop computer. Genome Res. 18(5), 802-809 (2008)

10. Chaisson, M., Pevzner, P., Tang, H.: Fragment assembly with short reads. Bioinformatics 20(13), 2067-2074 (2004)

11. Butler, J., et al.: ALLPATHS: de novo assembly of whole-genome shotgun microreads. Genome Res. 18(5), 810-820 (2008)

12. Pevzner, P.A., Tang, H., Waterman, M.S.: An Eulerian path approach to DNA fragment assembly. Proc. Natl. Acad. Sci. USA 98(17), 9748-9753 (2001)

13. Idury, R.M., Waterman, M.S.: A new algorithm for DNA sequence assembly. J. Comput. Biol. 2(2), 291-306 (1995)

14. Myers, E.W.: The fragment assembly string graph. Bioinformatics 21(suppl. 2), ii79-ii85 (2005)

15. Chin, F.Y., et al.: Finding optimal threshold for correction error reads in DNA assembling. BMC Bioinformatics 10(suppl. 1), S15 (2009)

16. http://www.ncbi.nlm.nih.gov/

\section{Appendix}

\section{A.1 Running Times of the Assembly Algorithms}

Figure 3 and Figure 4 show the running time of IDBA and existing assembly algorithms, Velvet, Abyss and Edena on the simulated data set and the real data set. From the figures, we can see that IDBA has similar running time as other assembly algorithms except Abyss which takes a very long time when $k$ is small due to a complicated method for removing dead-ends.

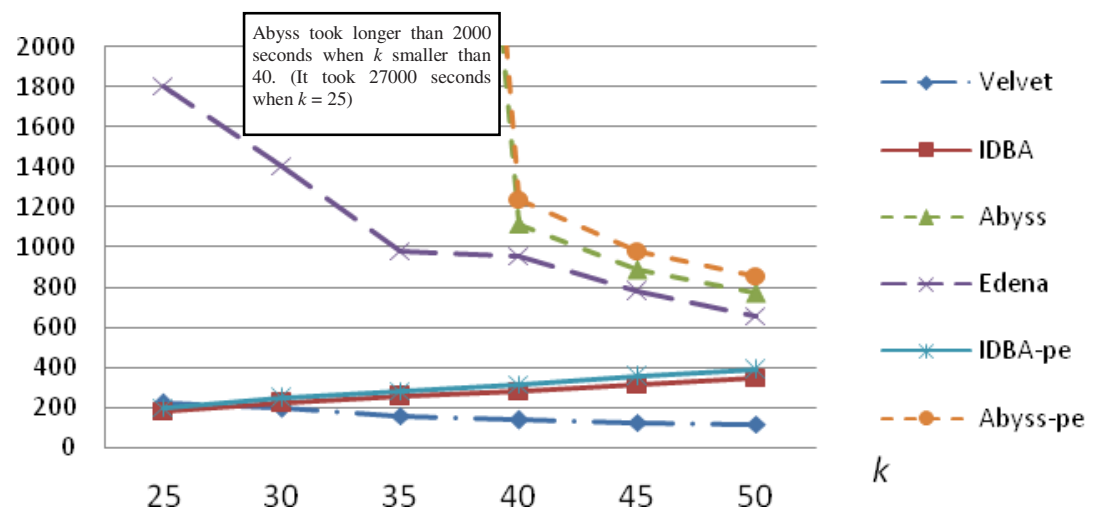

Fig. 3. Running time of assembly algorithms with different $k$ (or $x$ ) values on simulated data

\section{A.2 Detailed Comparison of the Assembly Algorithms for Real Data}

In Table 4, we show comprehensive statistics on the performance of the algorithms on their optimal $k$ value (w.r.t. N50) for the real dataset. IDBA produced much longer contigs than all other algorithms no matter whether the single-end or the pair-end version is used. The result is consistent with that of the simulated dataset. 


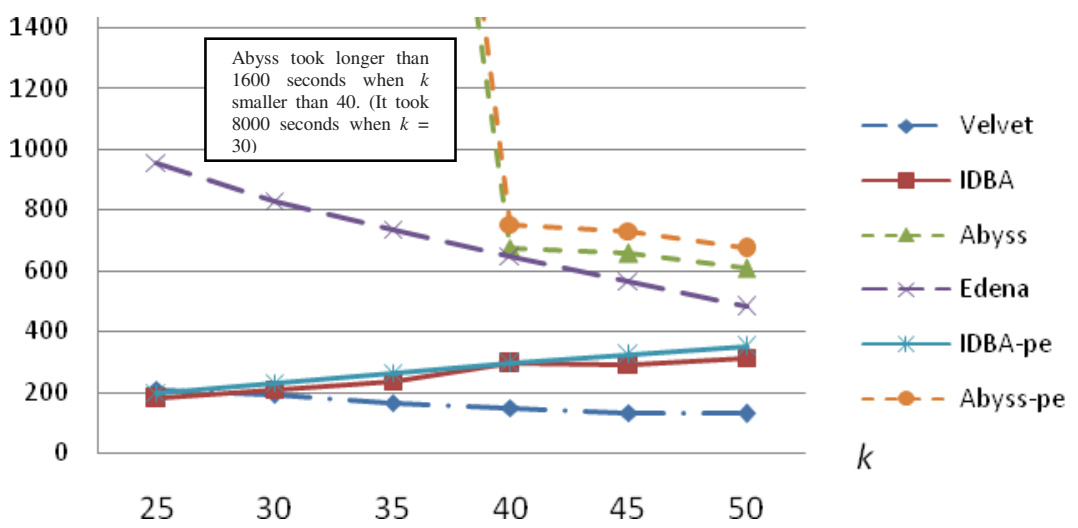

Fig. 4. Running time of assembly algorithms with different $k$ (or $x$ ) values on real data

Table 4. Statistics for the optimal (w.r.t. N50) result of each algorithm for real data

\begin{tabular}{lllllll}
\hline & \multirow{2}{*}{ Time } & \multirow{2}{*}{ Memory } & \multirow{2}{*}{$k$} & Total No. & N50 & Max length \\
\hline Velvet & $150 \mathrm{~s}$ & $893 \mathrm{M}$ & 40 & 335 & 57656 & 181399 \\
Edena & $649 \mathrm{~s}$ & $632 \mathrm{M}$ & 40 & 926 & 19423 & 66455 \\
IDBA & $325 \mathrm{~s}$ & $310 \mathrm{M}$ & $25-50$ & 267 & 140067 & 602412 \\
IDBA-pe & $361 \mathrm{~s}$ & $310 \mathrm{M}$ & $25-50$ & 203 & 187648 & 613166 \\
Abyss & $729 \mathrm{~s}$ & $923 \mathrm{M}$ & 40 & 445 & 30081 & 134067 \\
Abyss-pe & $3766 \mathrm{~s}$ & $936 \mathrm{M}$ & 35 & 406 & 120807 & 537397 \\
\hline
\end{tabular}

\section{A.3 Theorems and Proofs}

Theorem 3: Assume $m$ is the filtering threshold, the probability that a $k_{\min }-\operatorname{mer} v$ in the genome (except the first $l-k_{\min }$ and last $l-k_{\min } k_{\min }$-mer in the whole genome) does not appear in $H_{k_{\min }}$ (false negative) when $t$ length- $l$ reads are uniformly sampled from a length- $g$ genome with error rate $e$ is at most $\sum_{i=0}^{m}\left(\begin{array}{c}t \\ i\end{array}\right) p^{i}(1-p)^{t-i}$ where $p=\left[\left(l-k_{\min }+1\right) /(g-l+1)\right] \cdot(1-e)^{k_{\min }}$.

\section{Proof:}

$\operatorname{Pr}(v$ is sampled in a read $)$

$\operatorname{Pr}($ read contains $v$ is sampled $) \operatorname{Pr}(v$ is sampled $I$ read contains $v$ is sampled $)$

$=+\operatorname{Pr}($ read does not contain $v$ is sampled $) \operatorname{Pr}(v$ is sampled $I$ read does not contain $v$ is sampled $)$

$\geq \operatorname{Pr}($ read contains $v$ is sampled $) \operatorname{Pr}(v$ is sampled $I$ read contains $v$ is sampled $)$

$\geq \frac{l-k_{\min }+1}{g-l+1} \cdot(1-e)^{k_{\min }}$

The probability that a correct $k_{\min }$-mer $v$ appears no more than $m$ times is at most

$$
\sum_{i=0}^{m}\left(\begin{array}{l}
t \\
i
\end{array}\right) p^{i}(1-p)^{t-i} \text { where } p=\frac{l-k_{\min }+1}{g-l+1} \cdot(1-e)^{k_{\min }}
$$


Theorem 4: Assume that a contig $c$ in $H_{k}$ is treated as dead-end and removed. The probability that $c$ is a correct contig is less than

$$
2\left[1-\frac{l-k-2}{g-l+1} \cdot(1-e)^{k+3}\right]^{t}
$$

Proof: A contig $c$ in $H_{k}$ is treated as dead-end only if $c$ is of length less than $3 k-1$ and is not a dead end in $H_{k-1}$. Since all contigs in $H_{k-1}$ are preserved in $H_{k}, c$ is removed because (1) the length of $c$ is at least $3(k-1)-1$ and shorter than $3 k-1$, or (2) $c$ is shorter than $3(k-1)-1$ and one of its ends has 0 in-degree or out-degree in $H_{k}$. Thus $c$ will not be treated as a dead-end if the two adjacent $(k+3)$-mers of $c$ is sampled. By considering $k_{\min }=k+3$ and $m=0$ in Theorem 1 , the probability that no read contains a particular $(k+3)$-mer is at most

$$
\left[1-\frac{l-k-2}{g-l+1} \cdot(1-e)^{k+3}\right]
$$

and the probability that no read contains a particular $(k+3)$-mer cover a particular end of $c$ is at most

$$
2\left[1-\frac{l-k-2}{g-l+1} \cdot(1-e)^{k+3}\right]^{t}
$$

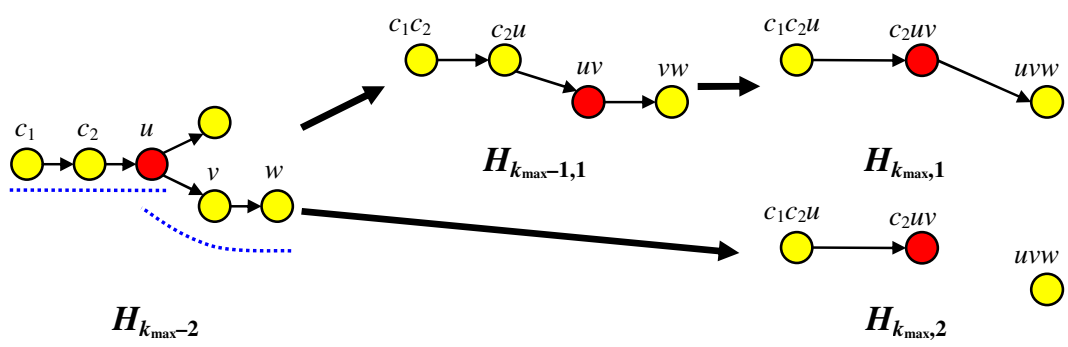

Fig. 5. Cases that a longer contig in $H_{k_{\max }, 1}$ does not exist in $H_{k_{\max , 2}}$

Let $H_{k, s}$ denote the accumulated de Bruijn graph $H_{k}$ with step size $s$. Theorem 5 shows that $H_{k_{\max }, 1}$ has at most the same number of gaps as $H_{k_{\max }, 2}$. There are some cases (Figure 5) that there is a longer contig in $H_{k_{\max }, 1}$ which is not in $H_{k_{\max }, 2}$. For example, consider a contig $c$ in $H_{k}$ which stops before vertex $u$ whose in-degree $=1$ and outdegree $>1$ and all branches of $u$ are shorter than $2 k$ and only $u$ to $v$ is correct. If there is only two reads contains $u$ and its 2 pairs of neighboring nucleotides at two ends respectively and there is no error read linking $c$ with other branches, there is a longer contig $c^{\prime}$ in $H_{k+2,1}$ that contains $c$ which does not appear in $H_{k+2,2}$.

Theorem 5: If a $k_{\max }$-mer $\left(k_{\max +1}\right.$-mer $)$ in the genome appears in $H_{k_{\max }, 2}$, it also appears in $H_{k_{\max }, 1}$. 
Proof: By induction on $k_{\max }$. Consider $k_{\max }=k_{\min }+2$. Given a $k_{\max }-\operatorname{mer}\left(\left(k_{\max }+1\right)\right.$-mer $)$ $v$ does not appear in $H_{k_{\max }, 1}$, let $v^{\prime}$ be the shortest substring of $v$ of length- $k$ which does not appear as a vertex in $H_{k, 1}$ or an edge in $H_{k-1,1}, k_{\min } \leq k \leq k_{\max }$.

Case 1: $k=k_{\min }$, i.e. $v$ ' does not appear in $H_{k_{\min }, 2}, v$ does not appear in $H_{k_{\max }+1,2}$.

Case 2: $k=k_{\min }+1$, there are two cases: (a) $v$ ' does not appear in $H_{k_{\min }, 1}$ as an edge or (b) $v^{\prime}$ is a vertex on a dead-end with length less than $2\left(k_{\min }+1\right)$ in $H_{k_{\min }+1,1}$. In case (a), since any $\left(k_{\min }+2\right)$-mer contains $v^{\prime}$ as substring does not appear in $H_{k_{\max }, 2}, v$ does not appear in $H_{k_{\max , 2}}$. In case (b), $v$ is a vertex on a dead-end with length less than $2\left(k_{\min }+2\right)$ in $H_{k_{\max }, 2}$ which will be removed.

Case 3: $k=k_{\min }+2$, there are two cases: (a) $v$ does not appear in $H_{k_{\min }+1,1}$ as an edge or (b) $v$ is a vertex on a dead-end with length less than $2\left(k_{\min }+2\right)$ in $H_{k_{\min }+2,1}$. In case (a), consider the path $\left(v_{1}, v_{2}, v_{3}\right)$ in $H_{k_{\min }, 1}$ representing the $\left(k_{\min }+2\right)$-mer $v$. Since $v$ does not appear in $H_{k_{\min }+1,1}$ as an edge, $v_{2}$ has $>1$ in-degree or out-degree and there is no read containing $v^{\prime}$ as subsring. Thus the in-degree and out-degree of $v$ are 0 in $H_{k_{\max }, 2}$ and $v$ will be removed as dead-end. In case (b), $v$ is a vertex on a dead-end with length less than $2\left(k_{\min }+2\right)$ in $H_{k_{\max , 2}}$ which will be removed.

Case 4: $k=k_{\min }+3$, i.e. $v$ does not appear in $H_{k_{\max }, 1}$ as an edge, the path $\left(v_{1}, v_{2}, v_{3}, v_{3}\right)$ in $H_{k_{\min }, 1}$ representing the $\left(k_{\min }+3\right)$-mer $v$ is not a potential contig and there is no read containing $v$ as a substring. Thus $v$ does not appear in $H_{k_{\max , 2}}$ as an edge. 